\title{
Developing a global cancer survivorship community: the Journal of Cancer Survivorship Social Media Site @jcansurv
}

\author{
Maryam B. Lustberg ${ }^{1} \cdot$ Larissa Nekhlyudov $^{2} \cdot$ Jennifer M. Jones ${ }^{3} \cdot$ Brad Love $^{4,5} \cdot$ Matthew S. Katz $^{6}$. \\ Michael Feuerstein ${ }^{7}$
}

Received: 5 March 2021 / Accepted: 11 March 2021 / Published online: 31 March 2021

(C) The Author(s), under exclusive licence to Springer Science+Business Media, LLC, part of Springer Nature 2021

As the number of cancer survivors continue to grow in the USA and globally, many individuals experience a range of acute, long-term, and late effects that can impact health, function, and well-being. The Journal of Cancer Survivorship: Research and Practice (JCSU) was launched in 2007 to promote dissemination of evidence-based cancer survivorship care throughout the world. The Journal has grown since its first issue, publishing original research, systematic, scoping, and meta-analytic reviews on topics that impact the quality of care and quality of life of cancer survivors.

In late 2019, the editorial team recognized an opportunity for the wider dissemination of the published papers in the Journal by communicating via a social media presence using the Twitter platform. Our intention was to expand awareness of the Journal's content for researchers, clinicians, cancer survivors, and other stakeholders. Venturing onto Twitter seemed timely given its growing and active scientific community, use of the service by multiple reputable journals, and mounting evidence that scholarly material presented or introduced on social media can increase uptake and impact of such knowledge [1].

Maryam B. Lustberg

maryam.lustberg@osumc.edu

1 Department of Medicine, Division of Medical Oncology, The Ohio State University, Columbus, OH, USA

2 Department of Medicine, Brigham and Women's Hospital, Harvard Medical School, Boston, MA, USA

3 Cancer Rehabilitation and Survivorship, Princess Margaret Cancer Centre, Toronto, ON, Canada

4 Center for Health Communication \& School of Advertising and Public Relations, University of Texas, Austin, TX, USA

5 Gryt Health, Rochester, NY, USA

6 Department of Radiation Medicine, Lowell General Hospital, Lowell, MA, USA

7 Gaithersburg, MD, USA
Additionally, social media presents an opportunity to increase survivor engagement and dissemination of accurate scientific information related to cancer survivorship [2].

The handle, @ jcansurv, went live on January 4, 2020, actively following and inviting cancer survivorship researchers, clinicians, cancer survivors, and organizations around the world to follow the account. The main activity of the site has been to post titles and brief summaries of papers published in the Journal. Access to articles in JCSU are provided by the SHAREit Link (https://www.ushareit.com) through our publisher, Springer Nature. Further dissemination of this work by followers is encouraged by tagging authors with other Twitter accounts. The @jcansurv handle is also followed by authors who post about their work and tag the journal in discussions of relevant topics in cancer survivorship.

Over the year since the launch, the account has grown with a current following of over 2000 individuals and organizations (Fig. 1). Although the geographic reach is global, North America and Australia represent the most active countries (Fig. 2). As can be seen in Fig. 3, most of the @ jcansurv followers are young and employed in health care occupations. (It should be noted that the accuracy of these data generated using the analytic platform tweetsmap (https://tweepsmap. com) have not independently vetted by @ jcansurv.) Engagement with the site has been variable (Fig. 4). While most followers indicated that they "liked" the material posted over the initial year, the number who retweeted posts to others was smaller.

In addition to highlighting Journal articles, the @ jcansurv has piloted other strategies over the past year to promote discussion and dissemination of current cancer survivorship issues. For example, @jcansurv collaborated with Cancer Survivor Social Media (@CancerSurvSM) and the Radiation Oncology Journal Club (\#radonc \#jc), on a series of Twittermoderated chats in May and April 2020. The first two chats were focused on engaging with cancer survivors on the impact 
2500

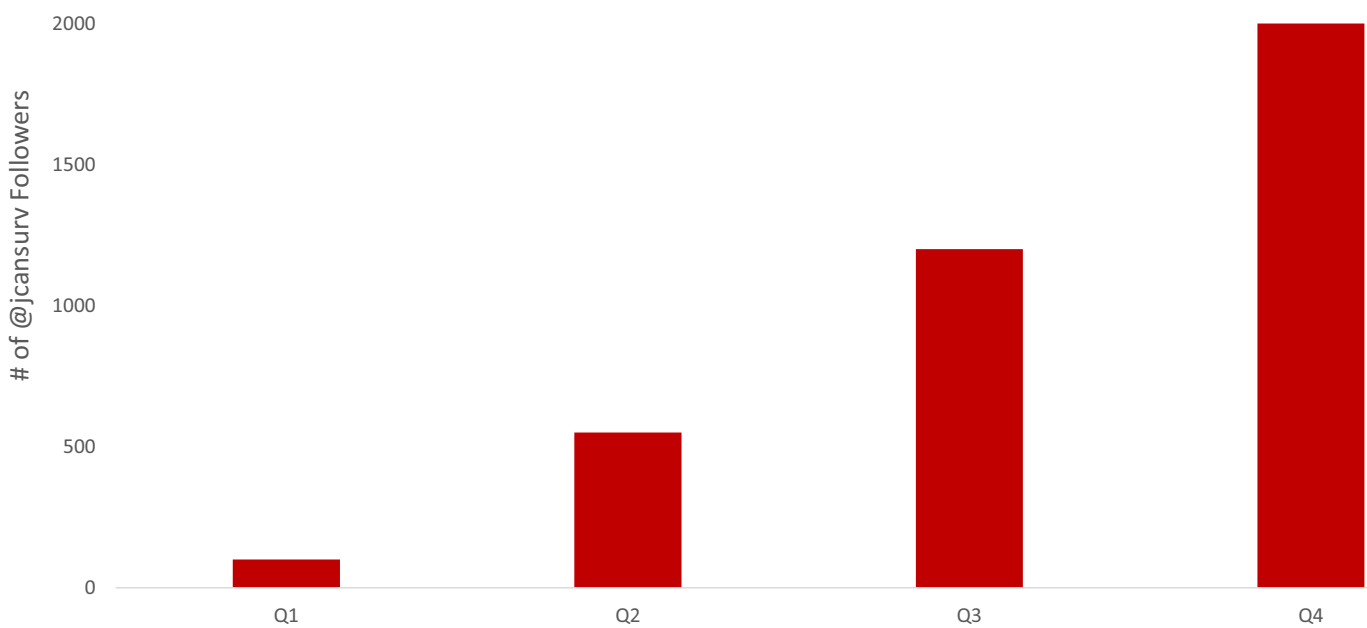

Fig. 1 Number of @ jcansurv followers in 2020 based on manual tracking by the handle social media editor

of COVID-19 and survivorship care [3] and reported on updated recommendations for the care of cancer survivors during the pandemic around the globe [4]. These events included clinicians, patients, and advocates sharing their experiences and insights on optimizing the care of cancer survivors during this challenging time. Another chat in August 2020 with Cancer Survivor Social Media focused on survivors living with incurable cancers. This discussion clearly demonstrated an interest in this topic within the cancer survivorship community. The paucity of coverage of this topic in the scientific literature was emphasized by attendees and further emphasized the need for our call for special section papers in the Journal, "Living with Incurable Cancer: Addressing Gaps in Cancer Survivorship" (https://www.springer.com/journal/ 11764). Through@jcansurv posts, we augmented recruitment of authors from around the world. This special section of the Journal will be published in 2021 .
Fig. 2 Global reach of @ jcansurv in 2020 based on data analytics site www.tweepsmap.com

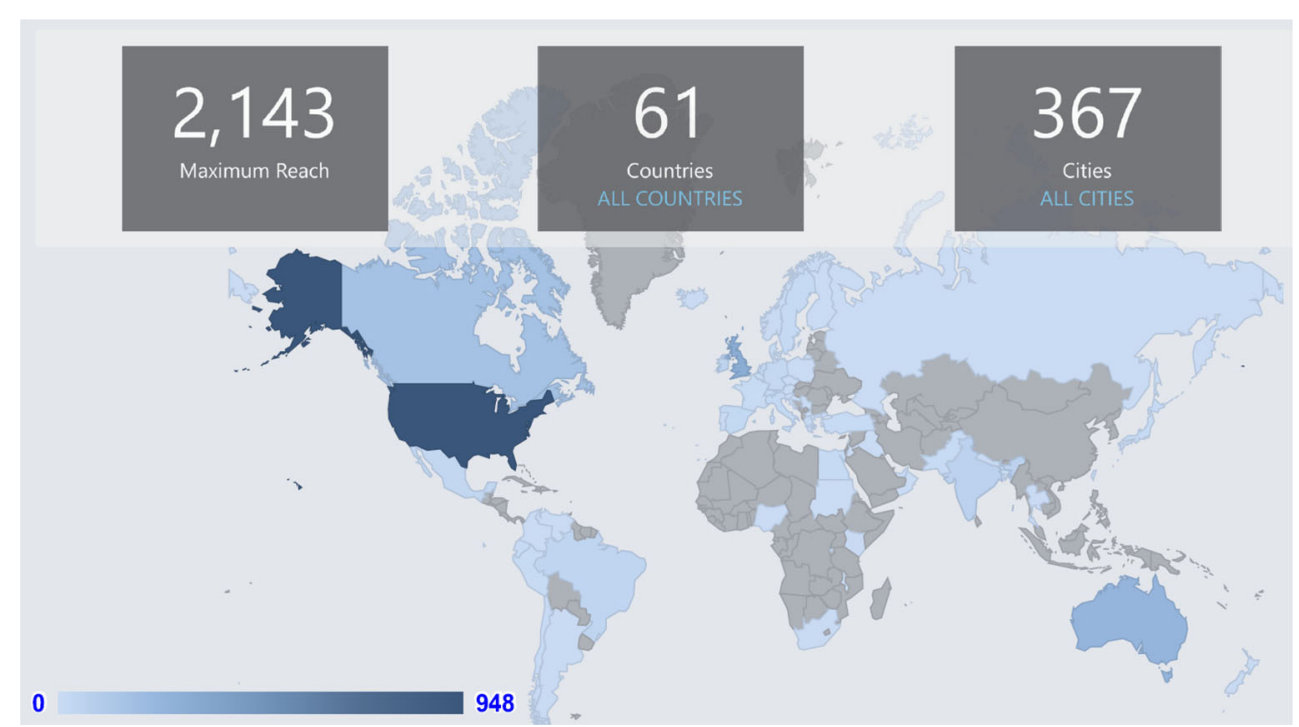


Fig. 3 Reported demographics of @ jcansurv followers in 2020 based on data analytics site from www.tweepsmap.com

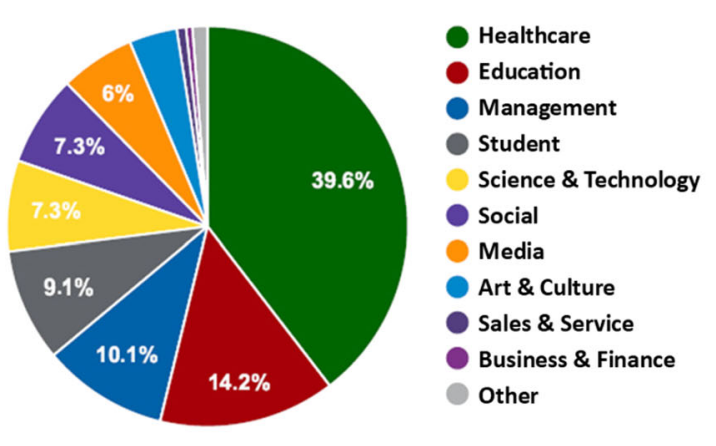

Female Male Businesses/ Groups
As part of the effort to facilitate communication on relevant topics in cancer survivorship research and practice, each post from@jcansurv also includes the hashtag \#survonc from Symplur (https://www.symplur.com) as part of the community building efforts to link with the diverse body of literature on cancer survivorship in social media.

In our first year, the @jcansurv hashtag has grown in recognition within the survivorship community and continues to be increasingly used for content from the Journal and other cancer survivorship related posts. To promote more regular interaction with the site, we plan to provide future communication efforts (e.g., podcasts or video conferences a few times per year). Additional efforts of @jcansurv will include the identification of new topics addressed in the Journal. This feedback loop can facilitate identification of relevant topic areas for future Special Sections and standard journal publications, thus improving the knowledge and application of innovative and timely research. The Journal is also considering interactive media including visual and audio abstracts as well as virtual mini-symposia on relevant topics in cancer survivorship. We anticipate that @jcansurv will continue to contribute as a connecting hub for the cancer survivorship community of researchers, clinicians, patient advocates, and survivors themselves to come together from around the world to tackle the many persistent challenges in both knowledge and care in cancer survivorship.

Expanding our global reach, with clinicians, researchers, cancer survivors, caregivers, and other stakeholders represents
Fig. 4 Communication trends in @ jcansurv in 2020 based on data analytics site www.tweepsmap. com
Tweets

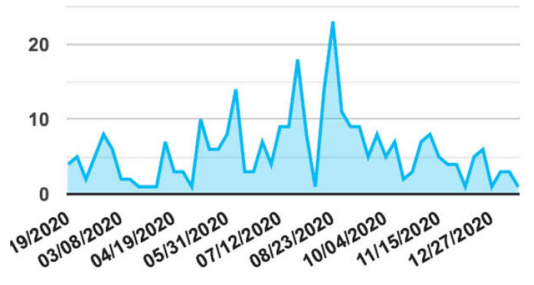

Engagements Timeline

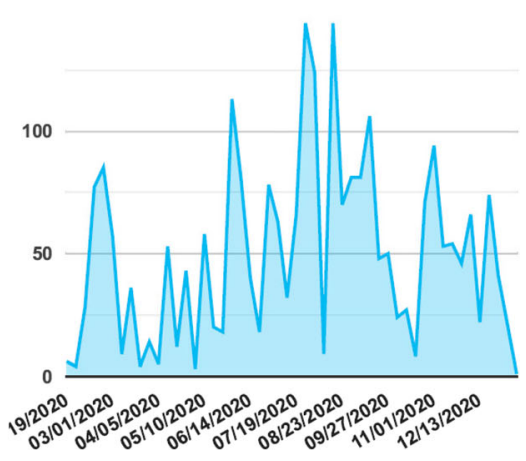


a goal for @jcansurv over the next few years. We plan to coordinate efforts with cancer survivorship organizations. The primary goal of establishing this social media outlet is to broaden the outreach of findings reported in the Journal of Cancer Survivorship: Research and Practice. We are well on our way and hope that our readers will take advantage of this effort.

\section{Declarations}

Conflict of interest Dr. Matthew Katz's relevant disclosures include Stock ownership: Dr. Reddy's, US Physical Therapy, Mazor Robotics, Healthcare Services Inc, and CVS Health. Dr. Brad Love has disclosure with the company Gryt Health. The other authors declare that they have no conflicts of interest. Dr. Michael Feuerstein is the founder and Editorin-Chief of the Journal of Cancer Survivorship.

\section{References}

1. Klar S, Krupnikov Y, Ryan JB, Searles K, Shmargad Y. Using social media to promote academic research: identifying the benefits of twitter for sharing academic work. PLoS One. 2020;15:e0229446.

2. Gentile D, Markham MJ, Eaton T. Patients with cancer and social media: harness benefits, avoid drawbacks. J Oncol Pract. 2018;14(12):731-7.

3. Nekhlyudov L, Duijts S, Hudson SV, Jones JM, Keogh J, Love B, et al. Addressing the needs of cancer survivors during the COVID-19 pandemic. J Cancer Surviv. 2020;14:601-6.

4. Jones JM, Saeed H, Katz MS, Lustberg MB, Forster VJ, Nekhlyudov L. Readdressingdressing the needs of cancer survivors during COVID-19: a path forward. J Natl Cancer Inst. 2020. https://doi. org/10.1093/jnci/djaa200.

Publisher's note Springer Nature remains neutral with regard to jurisdictional claims in published maps and institutional affiliations. 